\title{
Mutations in the Pseudomonas syringae avrRpt2 gene That Dissociate Its Virulence and Avirulence Activities Lead to Decreased Efficiency in AvrRpt2-Induced Disappearance of RIN4
}

\author{
Melisa T. S. Lim and Barbara N. Kunkel \\ Department of Biology, Washington University in St. Louis, St. Louis, MO 63130, U.S.A.
}

Submitted 29 May 2003. Accepted 20 October 2003.

\begin{abstract}
The avrRpt2 gene from Pseudomonas syringae pv. tomato exhibits avirulence activity on Arabidopsis expressing the resistance gene $R P S 2$ but promotes bacterial virulence on susceptible rps 2 Arabidopsis. To understand the functional relationship between the avirulence and virulence activities of avrRpt2, we analyzed a series of six avrRpt 2 mutants deficient in eliciting the RPS2-dependent hypersensitive response. We show that the mutants are also severely impaired in triggering $R S P 2$-dependent resistance. Four of these mutants are severely impaired in their virulence activity, whereas two alleles, encoding C-terminal deletions of AvrRpt2, retain significant but slightly reduced virulence activity. Thus, the avirulence and virulence activities of avrRpt 2 can be genetically uncoupled. We tested the ability of the two C-terminal deletion mutants to trigger AvrRpt2induced elimination of the Arabidopsis RIN4 protein and show that they retain this activity but are less efficient than wild-type AvrRpt2. Thus, reduced AvrRpt2 virulence activity is correlated with reduced efficiency in the induction of RIN4 disappearance. This suggests that an alteration in kinetics of RIN4 disappearance triggered by the C-terminal deletion mutants may provide the mechanistic basis for the uncoupling of the avirulence and virulence activities of avrRpt2.
\end{abstract}

Additional keywords: avrRpm1, RPM1.

To resist pathogen attack, plants have evolved the ability to recognize and respond to specific pathogens by mounting defense responses that serve to limit pathogen growth and spread. In many cases, the ability to trigger disease resistance is determined by specific pathogen avirulence (avr) genes whose presence is recognized by plants expressing cognate host resistance $(R)$ genes (Dangl and Jones 2001). Given that avr genes render pathogens unable to cause disease on resistant hosts, their maintenance by pathogens suggests that these genes must confer a selective advantage in certain situations. Accordingly, several avr genes initially identified based on their avirulence activities have been shown to contribute to pathogen virulence on susceptible host plants lacking the corresponding $R$ gene (Leach et al. 2001).

An example of a well-characterized $a v r$ gene possessing both avirulence and virulence activities is avrRpt2 from Pseudomonas syringae pv. tomato, the causal agent of bacterial

Corresponding author: B. N. Kunkel; E-mail: Kunkel@biology.wustl.edu speck disease on tomato. avrRpt 2 was identified based on its ability to trigger resistance on Arabidopsis lines harboring the cognate $R$ gene, RPS2 (Kunkel et al. 1993; Whalen et al. 1991; Yu et al. 1993). Thus, $P$. syringae carrying avrRpt 2 causes significantly less severe disease on Arabidopsis plants expressing RPS 2 than does $P$. syringae lacking avrRpt2. This dramatic reduction in severity of disease symptoms is paralleled by a significant reduction in bacterial growth over the course of infection (Kunkel et al. 1993; Yu et al. 1993). As is common with $R$-gene-mediated resistance, one of the defense responses associated with RPS2-mediated resistance is the hypersensitive response (HR), a form of localized cell death that occurs at the site of infection (Greenberg 1997).

In the absence of a functional RPS 2 gene, avrRpt 2 promotes the ability of $P$. syringae to grow and cause disease. Thus, on susceptible lines of Arabidopsis, avrRpt 2 functions as a virulence gene (Chen et al. 2000; Guttman and Greenberg 2001). The increase in virulence conferred by avrRpt 2 is most readily detectable on Arabidopsis lines that exhibit reduced susceptibility to $P$. syringae, such as ecotype Nossen (No-0) (Chen et al. 2000). avrRpt2 can also promote the virulence of $P$. syringae strains expressing the avrRpml avirulence gene by interfering with resistance and the HR triggered by this strain on Arabidopsis expressing the cognate RPM1 gene (Ritter and Dangl 1996). Although avrRpt2 has the ability to enhance the virulence of $P$. syringae in these two distinct situations, the functional relationship between the two virulence activities remains unclear (Chen et al. 2000).

Several lines of evidence indicate that AvrRpt2 functions from within the plant cell to exert both its virulence and avirulence effects. When expressed by $P$. syringae, avrRpt 2 activity is dependent on the function of the bacterial type III secretion system (TTSS) (Mudgett and Staskawicz 1999), an apparatus used to secrete and translocate bacterial protein effectors directly into the host cell (Galan and Collmer 1999). Transient expression of AvrRpt2 in Arabidopsis leaves is sufficient to trigger localized cell death in an RPS2-dependent manner (Leister et al. 1996; McNellis et al. 1998). Transgenic Arabidopsis plants lacking a functional RPS2 gene that constitutively express avrRpt2 exhibit enhanced susceptibility to $P$. syringae pv. tomato DC3000 infection and are unable to mount avrRpml and RPMI-mediated resistance (Chen et al. 2000). Furthermore, AvrRpt 2 protein is effective in triggering RPS2-dependent cell death in Arabidopsis protoplasts only in the presence of a carrier protein that allows AvrRpt 2 to enter the cell (Wu et al. 2003). 
The mode of action of AvrRpt2 is not well understood. avrRpt 2 encodes a $28.2-\mathrm{kDa}$ protein whose primary amino acid sequence provides no obvious clues regarding its biochemical function. Recently, based on an alignment of the predicted secondary structure, AvrRpt 2 has been suggested to be a cysteine protease (Axtell et al. 2003). Further insight into the mode of action of AvrRpt 2 comes from the recent observation that AvrRpt 2 has the ability to induce the rapid disappearance of RIN4, an Arabidopsis protein that interacts with both RPM1 and AvrRpm1 (Mackey et al. 2002). Plants with reduced levels of RIN4 are compromised for both RPMI-mediated resistance and induction of the HR in response to $P$. syringae expressing AvrRpm1 (Mackey et al. 2002). These results suggest that the AvrRpt2-induced disappearance of RIN4 is the mechanism by which AvrRpt 2 interferes with RPM1-mediated responses in Arabidopsis. Further evidence suggests that the avirulence activity of AvrRpt 2 may also be mediated through RIN4. RIN4 was shown to interact with RPS2 (Axtell and Staskawicz 2003; Mackey et al. 2003). Strikingly, the overexpression of RIN4 inhibits RPS2-mediated resistance in response to infection by $P$. syringae pv. tomato expressing avrRpt 2 , while the lethality of a rin4 null mutant is rescued when the mutation is

A
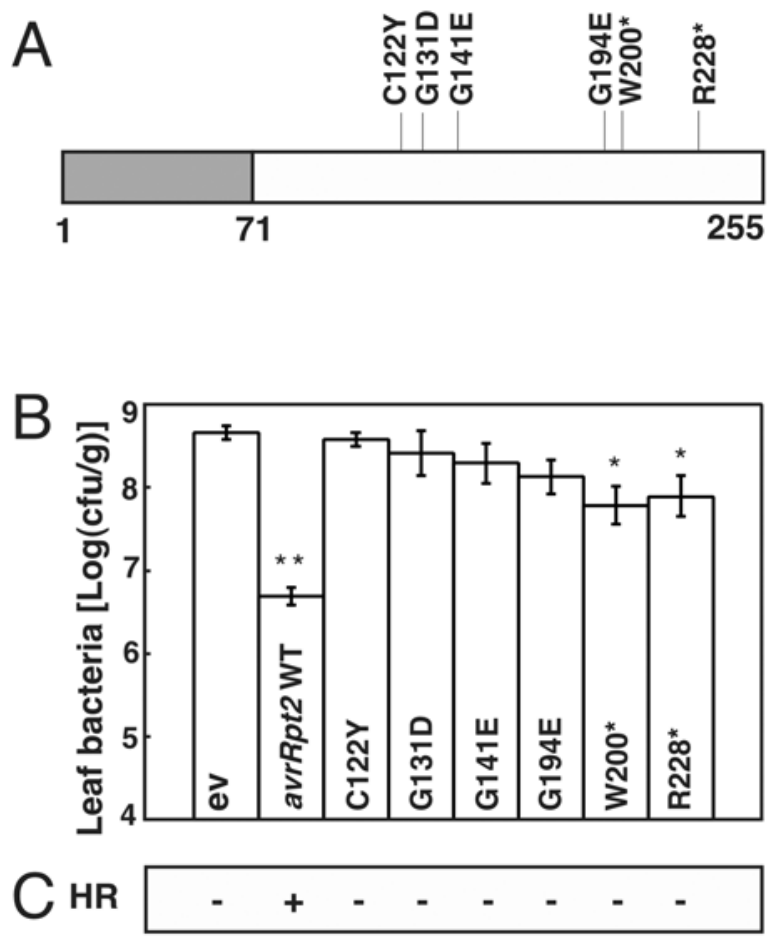

Fig. 1. Mutations that disrupt the avirulence function of avrRpt2. A, Schematic of the AvrRpt 2 protein showing the position of mutations introduced individually by site-directed mutagenesis. The grey area shows the N-terminal 71 amino acids that are cleaved when AvrRpt2 is inside the plant. B, Growth of Pseudomonas syringae pv. tomato DC3000 carrying either an empty vector (ev), wild-type (WT) avrRpt2, or the indicated avrRpt 2 mutant alleles in Arabidopsis Col-0 RPS2. Plants were inoculated by dipping them into bacterial suspensions $\left(1 \times 10^{6} \mathrm{CFU} / \mathrm{ml}\right)$, and the total number of leaf bacteria was measured 4 days after inoculation. The number of bacteria measured $1 \mathrm{~h}$ after inoculation was similar across all treatments (data not shown). Error bars represent the standard error among three samples. Asterisks indicate statistically significant differences of means compared with the P. syringae pv. tomato DC3000 (ev) treatment (Student's $t$ test, $* P<0.05,{ }^{* *} P<0.01$ ). Similar results were obtained in three independent growth experiments. C, Ability of $P$. syringae pv. tomato DC3000 carrying either an empty vector (ev), wildtype (WT) avrRpt2, or the mutants to elicit a hypersensitive response (HR) on Arabidopsis Col-0 RPS2. Leaves were scored for presence (+) or absence (-) of tissue collapse at $18 \mathrm{~h}$ postinoculation. HR results are summarized from three independent experiments. crossed into an rps 2 mutant background (Mackey et al. 2003). These results have been incorporated into a model in which the AvrRpt2-induced disappearance of RIN4 leads to the activation of RPS2-dependent defenses (Axtell and Staskawicz 2003; Mackey et al. 2003).

This model fits well with the so-called "guard hypothesis" (Dangl and Jones 2001; Van Der Biezen and Jones 1998), which postulates that the cellular virulence targets of bacterial TTSS effectors are guarded by $R$ gene products that monitor the status of the associated targets. Upon detecting the modification of the associated target by a TTSS effector, the $R$ gene product activates downstream defense responses leading to resistance. Hence it has been proposed that RIN4 forms part of a defense-activating complex that is targeted by AvrRpt 2 in an attempt by the pathogen to enhance host susceptibility and that the function of RPS2 in resistance is to monitor the status of that complex (Mackey et al. 2003). One prediction of this model is that the avirulence and virulence activities of AvrRpt 2 should be tightly coupled, since the removal of RIN4 is hypothesized to be the molecular basis of both activities. However, the role of RIN4 in defense or in disease development is unclear. Moreover, the role of RIN4 disappearance in the ability of AvrRpt 2 to enhance the virulence of $P$. syringae on susceptible Arabidopsis has not been examined.

We are interested in understanding the functional relationship between the avirulence and virulence activities of avrRpt2. To explore this question, we took advantage of a series of known mutations reported to abolish the ability of avrRpt2 to trigger a RPS2-dependent HR on Arabidopsis (Axtell et al. 2001). We extended the analysis of a subset of six of these mutations by examining their effect on the virulence activity of avrRpt2 on susceptible hosts. We show that two of the alleles, carrying nonsense mutations that result in C-terminal deletions of AvrRpt2, are severely impaired in their avirulence activity but retain significant levels of virulence activity. Thus, the avirulence and virulence activities of AvrRpt 2 can be genetically uncoupled. We analyzed the effect of the six AvrRpt2 mutants on RIN4 and show that virulence activity is correlated with the efficiency with which the mutants trigger RIN4 disappearance. We hypothesize that a differential requirement for AvrRpt2-induced RIN4 elimination over the course of infection provides the mechanistic basis for the uncoupling of the virulence and avirulence activities of AvrRpt2

\section{RESULTS}

\section{AvrRpt 2 mutants defective in eliciting RPS2-dependent cell death are also impaired in triggering RPS2-mediated disease resistance in Arabidopsis.}

Using a transgenic Arabidopsis line expressing avrRpt2 under a dexamethasone (DEX)-inducible promoter to screen for mutants defective in RPS2-mediated cell death, Axtell and associates (2001) isolated several Arabidopsis lines carrying mutations in the avrRpt 2 transgene. When expressed in $P$. syringae pv. tomato DC3000, the mutant avrRpt2 alleles failed to induce an RPS2-dependent HR on Arabidopsis, suggesting that they were compromised in their avirulence activity. To determine the effect of these mutations on the virulence activity of avrRpt2, we introduced a subset of these mutations (Fig. 1A) individually into the avrRpt2 gene carried on pVSP61, a stable broad-host-range vector that allows expression of avrRpt 2 virulence activity (Chen et al. 2000). To confirm that the introduced mutations also abolished avrRpt 2 avirulence activity when carried on pVSP61, we tested the ability of $P$. syringae pv. tomato DC3000 expressing these mutant alleles to trigger RPS2-mediated defenses. Consistent with the results of Axtell and associates (2001), $P$. 
syringae pv. tomato DC3000 strains expressing the newlygenerated mutant alleles failed to elicit a HR on wild-type Arabidopsis plants from accessions Columbia (Col-0) and No-0, both of which carry a functional RPS2 gene (Fig. 1C). Interestingly, mutations $\mathrm{C} 122 \mathrm{Y}$ and G131D had initially been identified in the same avrRpt2 transgene in a single transgenic Arabidopsis line (allele C122Y/G131D) (Axtell et al. 2001). However, when introduced separately into avrRpt2, both abolished the RPS2-dependent HR (Fig. 1C). To test the ability of the mutant alleles shown in Figure 1A to elicit an effective RPS2-mediated resistance response, we assayed the ability of $P$. syringae pv. tomato DC3000 expressing the avrRpt 2 mutant genes to grow and to cause disease on Arabidopsis. Col-0 plants infected with $P$. syringae pv. tomato DC3000 expressing wild-type avrRpt 2 mount an effective resistance response and thus do not develop disease symptoms (Kunkel et al. 1993). In contrast, Col-0 plants infected with strains carrying the mutant avrRpt 2 alleles developed disease symptoms indistinguishable in their severity from those produced by $P$. syringae pv. tomato DC3000 carrying the empty pVSP61 vector (data not shown). While $P$. syringae pv. tomato DC3000 carrying wild-type avrRpt2 grew to levels 50to 100 -fold lower than $P$. syringae pv. tomato DC3000 carrying pVSP61, strains carrying the mutant avrRpt 2 alleles grew to similar levels as P. syringae pv. tomato DC3000 (Fig. 1B). Thus, when expressed in $P$. syringae pv. tomato DC3000, the avrRpt 2 mutant alleles shown in Figure 1A are severely impaired in their ability to elicit RPS2-specific resistance, suggesting that they have lost avirulence activity. Interestingly, in some experiments, we observed that expression of the two nonsense mutant alleles W200* and R228* that encode Cterminal deletions of AvrRpt 2 resulted in slightly reduced growth on Col-0 as compared with $P$. syringae pv. tomato DC3000.

\section{Two avrRpt 2 mutants with impaired}

avirulence activity retain virulence activity.

We next assayed the virulence activity of the avrRpt2 mutant alleles on susceptible Arabidopsis plants lacking a functional RPS 2 gene. Wild-type avrRpt 2 dramatically enhances the ability of $P$. syringae pv. tomato DC3000 to grow and cause disease on No-0 rps 2 plants (Fig. 2) (Chen et al. 2000). P. syringae pv. tomato DC3000 carrying the four avrRpt 2 missense alleles did not reproducibly grow to higher levels or produce more severe disease symptoms than $P$. syringae pv. tomato DC3000 (Fig. 2). In contrast, P. syringae pv. tomato DC3000 carrying the W200* and R228* nonsense alleles consistently grew to higher levels on No-0 rps 2 plants than did $P$. syringae pv. tomato DC3000, often to levels similar to those achieved by $P$. syringae pv. tomato DC3000 carrying wild-type avrRpt2 (Fig. 2A). Infection of No-0 rps 2 plants with $P$. syringae pv. tomato DC3000 results in mild disease symptoms consisting of few to no individual water-soaked lesions (IWSL) on younger leaves and the sporadic appearance of large water-soaked patches (WSP) and chlorosis on older leaves (Fig. 2C). Expression of wild-type avrRpt2 in P. syringae pv. tomato DC3000 results in a dramatic increase in the frequency and severity of all of these symptoms (Fig. 2B) (Chen et al. 2000). Infection of No-0 rps 2 plants with $P$. syringae pv. tomato DC3000 expressing either of the two nonsense alleles also resulted in increased symptom development compared with $P$. syringae pv. tomato DC3000 alone (Fig. 2B). However, these strains consistently produced fewer WSP and IWSL than $P$. syringae pv. tomato DC3000 expressing wildtype avrRpt2 (Fig. 2B), indicating that the two nonsense mutations result in a reduction in $a v r R p t 2$ virulence activity. Thus, the W200* and R228* alleles that are severely impaired in their ability to trigger RPS2-mediated resistance retain significant virulence activity on susceptible Arabidopsis plants and appear to functionally uncouple the avirulence and virulence activities of avrRpt 2 .
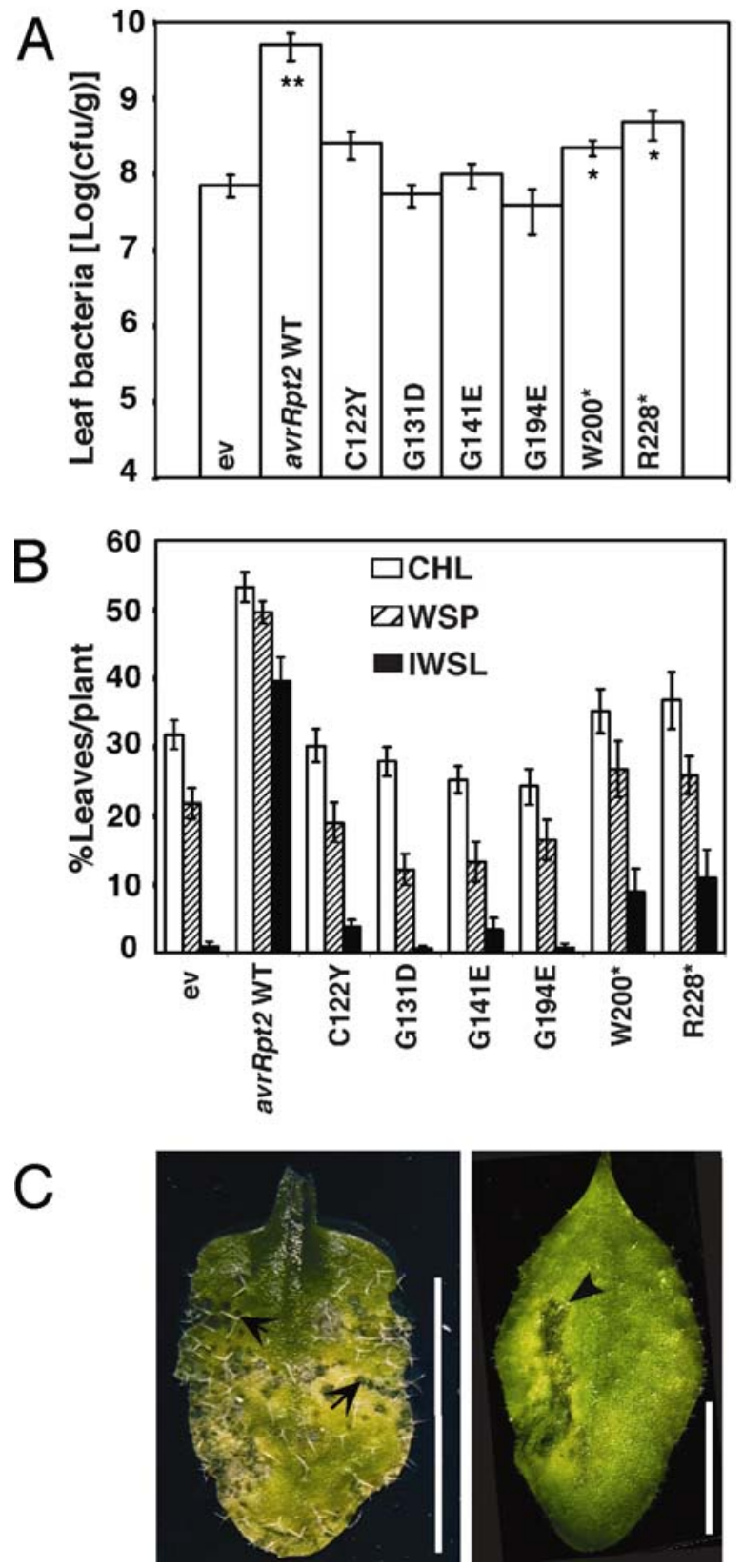

Fig. 2. Two C-terminal deletion mutants of AvrRpt 2 retain virulence activity. Measurements of growth and disease produced by Pseudomonas syringae pv. tomato DC3000 carrying the empty vector (ev), wild-type (WT) avrRpt2, or the indicated avrRpt2 mutant alleles on Arabidopsis No-0 rps2. Plants were inoculated by dipping them into bacterial suspensions $\left(2 \times 10^{8} \mathrm{CFU} / \mathrm{ml}\right)$, and measurements were made at 4 days after inoculation. A, Total number of leaf bacteria. Error bars represent the standard error among three samples. Asterisks indicate statistically significant differences of means compared with the $P$. syringae pv. tomato DC3000 (ev) treatment (Student's $t$ test, $* P<0.1,{ }^{*} P<<0.01$ ). Similar results were obtained from at least three independent growth experiments. B, Quantification of disease symptom formation. Leaves from single plants were individually scored for presence of chlorosis (CHL), water-soaked patches (WSP), and individual water-soaked lesions (IWSL) (shown in C). Bars represent average percentages of leaves per plant exhibiting these symptoms. A total of 12 plants were examined for each strain. Error bars represent standard error. Similar results were obtained from three independent experiments. C, Leaf exhibiting typical symptoms consisting of WSP (right; arrowhead) and IWSL (left; arrows). Bar $=5 \mathrm{~mm}$. 


\section{Mutant AvrRpt2 proteins}

do not undergo detectable host-dependent processing.

The absence of both the avirulence and virulence activities of the four missense avrRpt 2 mutants prompted us to analyze the effect of the mutations on stability of the mutant proteins in plant cells. Axtell and associates (2001) demonstrated that, in their system, avrRpt 2 alleles carrying all six mutations produced stable protein when expressed in P. syringae pv. tomato DC3000 cells grown in culture. However, given that AvrRpt2 functions within the plant cell to exert both its virulence and avirulence activities (Chen et al. 2000; Leister et al. 1996), we wanted to examine the stability of the mutant AvrRpt 2 proteins in plant tissue after delivery by $P$. syringae pv. tomato DC3000. In an attempt to distinguish AvrRpt2 present inside bacterial cells from protein that has been translocated into plant cells, we took advantage of the observation that the 28.2$\mathrm{kDa}$ AvrRpt2 protein is processed into a $20.7-\mathrm{kDa}$ C-terminal polypeptide when present inside plant cells (McNellis et al. 1998; Mudgett and Staskawicz 1999). We assayed the steadystate levels of AvrRpt 2 in total protein extracts from plants infected with $P$. syringae pv. tomato DC3000 expressing either wild-type or mutant avrRpt2. In the case of all the mutants, with the exception of the G194E allele, the unprocessed form of AvrRpt 2 was readily detectable in the infected plants (Fig. 3A). This indicates that, in our expression system, all of the mutant alleles of avrRpt 2 except for G194E express stable protein in $P$. syringae. However, despite the presence of high levels of full-length protein, the processed form of AvrRpt2
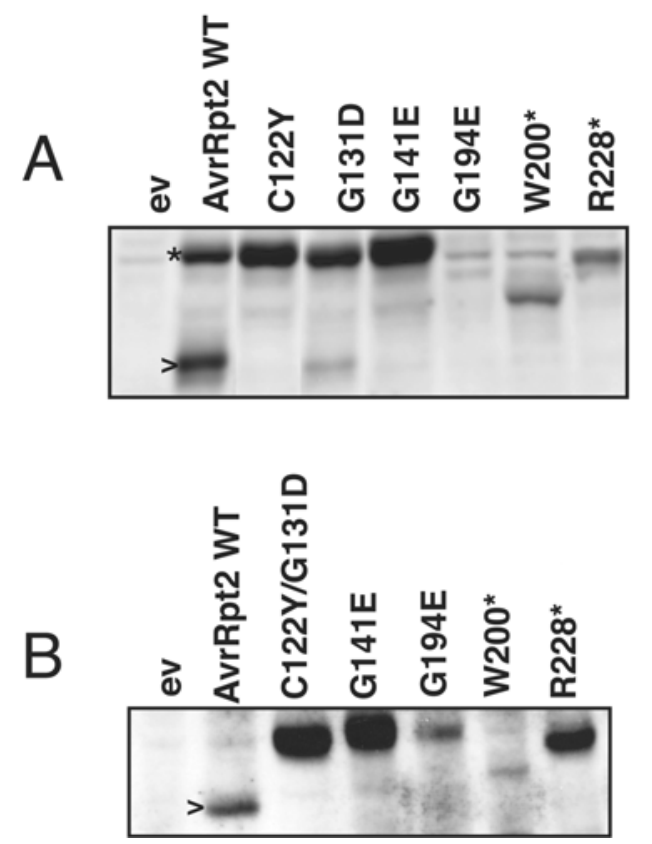

Fig. 3. Expression of proteins encoded by the mutant avrRpt2 alleles. A, AvrRpt2 protein expression during infection of Arabidopsis by Pseudomonas syringae pv. tomato DC3000 carrying an empty vector (ev), wild-type (WT), or the mutant avrRpt2 alleles. Arabidopsis Col-0 rps 2 leaves were syringe-inoculated with bacterial suspensions of $5 \times 10^{8}$ $\mathrm{CFU} / \mathrm{ml}$, and leaves were harvested $6.5 \mathrm{~h}$ after inoculation. B, AvrRpt2 protein expression in planta under the control of a dexamethasone (DEX)inducible promoter. Leaves of transgenic plants were infiltrated with 25 $\mu \mathrm{M}$ DEX and were harvested at $3 \mathrm{~h}$ postinoculation. Blots of total protein extracts from treated plants were reacted with anti-AvrRpt2 antibody. The location of the 28.2-kDa full-length and $20.7-\mathrm{kDa}$ processed form of AvrRpt 2 are indicated by $*$ and $>$, respectively. The truncated W200* and R228* mutant proteins are predicted to be $21.8 \mathrm{kDa}$ and $24.8 \mathrm{kDa}$, respectively. The processed form of these mutant proteins, predicted to be $14.3 \mathrm{kDa}$ for $\mathrm{W} 200^{*}$ and $17.3 \mathrm{kDa}$ for R228*, were not detectable in the expected size range on protein blots (data not shown). These experiments were repeated three times with similar results. was not readily detectable in extracts of plant tissue infected with $P$. syringae pv. tomato DC3000 carrying either of the six mutant alleles (Fig. 3A). The processed form of AvRpt 2 was detectable in plants infected with $P$. syringae pv. tomato DC3000 expressing the G131D mutant in some experiments (for example, in Fig. 3A), but the amount of processed protein was variable and was always significantly less than that detected in plants infected with P. syringae pv. tomato DC3000 expressing wild-type AvrRpt 2 .

Three plausible explanations could account for the absence of detectable processed AvrRpt2 in tissue infected with $P$. syringae pv. tomato DC3000 expressing the mutant alleles of avrRpt2. First, it is possible that the mutant proteins are not translocated into the plant cell and are thus not processed. Second, they may be translocated but fail to be processed. Lastly, the proteins may be translocated and processed within the plant tissue, but the processed forms may be unstable in planta. In an attempt to distinguish between these possibilities, we examined the levels of AvrRpt 2 expressed in transgenic Arabidopsis lines carrying the avrRpt 2 mutant alleles under the control of a DEX-inducible promoter (Axtell et al. 2001; McNellis et al. 1998). Consistent with the observations of McNellis and associates (1998), following DEX treatment, only the processed form of AvrRpt2, a protein in the range of $20 \mathrm{kDA}$, was detected in Arabidopsis plants expressing wildtype avrRpt2 (Fig. 3B). Surprisingly, only full-length AvrRpt2 was detectable in the lines expressing mutant avrRpt2 alleles (Fig. 3B). This result demonstrates that the mutant forms of AvrRpt 2 can be stably expressed inside the plant cell but that they are either not efficiently processed or that the processed forms of the mutant proteins are not stable inside plant cells. Consistent with the observed instability of the G194E protein in P. syringae, we also observed reduced levels of this mutant protein in transgenic plant lines expressing this allele (Fig. 3B).

Therefore, because the processed forms of AvrRpt 2 encoded by the C122Y, G131D, and G141E alleles are not reproducibly detectable in plant cells, we cannot determine whether these mutant proteins are translocated into the plant cell upon infection by $P$. syringae pv. tomato DC3000. Interestingly, the processed forms of AvrRpt 2 encoded by the W200* and R228* mutant alleles were also not detectable in planta (Fig. 4B). However, given that these two alleles retain virulence activity (Fig. 2) and the ability to induce RIN4 disappearance (discussed below), we deduce that the proteins encoded by these alleles can be translocated from $P$. syringae pv. tomato DC3000 into the host cell during infection.

\section{AvrRpt 2 alleles that maintain virulence activity exhibit a delay in inducing the disappearance of RIN4.}

Delivery of wild-type AvrRpt 2 by $P$. syringae pv. tomato DC3000 induces the disappearance of RIN4 in infected plants (Axtell and Staskawicz 2003; Mackey et al. 2003). In contrast, P. syringae pv. tomato DC3000 expressing alleles of avrRpt2 with either of the G121D/C122Y, G141E, or C194E mutations fails to trigger both the RPS2-dependent HR and the disappearance of RIN4 (Axtell and Staskawicz 2003; Axtell et al. 2001). These results support the hypothesis that the AvrRpt2induced reduction in RIN4 protein levels leads to the activation of RPS2-mediated resistance. Since AvrRpt 2 presumably first evolved as a virulence factor, RIN4 has been proposed to be a component of a defense-activating complex that is targeted for inactivation by AvrRpt2 (Mackey et al. 2003). However, the role of RIN4 elimination in the virulence activity of AvrRpt2 has not been addressed. To begin to explore the relationship between the virulence activity of AvrRpt 2 and RIN4 disappearance, we tested the ability of mutant AvrRpt 2 proteins en- 
coded by the W200* and R228* alleles to induce RIN4 elimination in infected plants. As controls, we included in our assays the missense alleles that have been reported to lack the ability to induce RIN4 disappearance (Axtell and Staskawicz 2003).

Consistent with the results of Axtell and associates(Axtell et al. 2001), when delivered by $P$. syringae pv. tomato DC3000, wild-type AvrRpt 2 induced a significant reduction in RIN4 levels by $6 \mathrm{~h}$ postinoculation, but the mutant proteins encoded by alleles C122Y, G131D, G141E, and G194E did not (Fig. 4A). Since the W200* and R228* mutants are severely impaired in activating RPS2-dependent resistance responses, we expected that they would also have lost the ability to trigger RIN4 disappearance. Surprisingly, the expression of these alleles reproducibly led to a significant reduction in levels of RIN4 in infected tissue by $6 \mathrm{~h}$ postinoculation (Fig. 4A). This finding seemed inconsistent with the model of RIN4-mediated activation of $R P S 2$ resistance.

One possible explanation for this apparent discrepancy was that the two C-terminal deletion mutants of AvrRpt 2 retain the ability to trigger both RIN4 elimination and RPS2-dependent defenses but do so with reduced efficiency when compared with wild-type AvrRpt2. To test this hypothesis, we carried out a more detailed analysis of the timing of RIN4 disappearance in Arabidopsis plants infected with $P$. syringae pv. tomato DC3000 expressing the W200* and R228* mutants. To increase the likelihood of detecting subtle changes in the kinetics of RIN4 disappearance, we attempted to slow down the rate of RIN4 disappearance by reducing the total amount of AvrRpt2 delivered to the plant at the beginning of infection. Therefore, we decreased the inoculum of bacteria to 10 times lower than that used in the experiment shown in Figure 4A. At this inoculum, a significant reduction in RIN4 levels was detectable in plants infected with $P$. syringae pv. tomato DC3000 expressing wild-type avrRpt 2 within 4 to $5 \mathrm{~h}$ postinoculation (Fig. 4B). However, reduction in RIN4 levels was delayed in plants infected with $P$. syringae pv. tomato DC3000 expressing the mutant alleles. A reduction in RIN4 level was detectable 6 to $9 \mathrm{~h}$ after plants were infected with strains expressing the W200* allele and not until $24 \mathrm{~h}$ after plants were infected with $P$. syringae pv. tomato DC3000 expressing the R228* allele (Fig 4B). Taken together, these results indicate that the AvrRpt2 mutant proteins encoded by the two nonsense alleles retain the ability to induce RIN4 disappearance but are less efficient in doing so than wild-type AvrRpt2.

Thus, the four AvrRpt 2 missense mutants that do not exhibit detectable virulence activity also fail to trigger the disappearance of RIN4, while the two nonsense mutants that maintain virulence activity retain the ability to trigger the disappearance of RIN4. Furthermore, the two nonsense mutations result in a delay in the AvrRpt2-induced disappearance of RIN4, and this delay is correlated with a reduction in virulence activity.

\section{C-terminal deletion mutants}

\section{of AvrRpt 2 trigger RPS2-dependent cell-death}

when expressed at high levels in planta.

The observation that the two C-terminally truncated AvrRpt 2 proteins encoded by the W200* and R228* mutants are unable to elicit RPS2-dependent defenses is inconsistent with the hypothesis that the elimination of RIN4 activates RPS2-dependent defense pathway. Instead, this hypothesis leads to the prediction that the W200* and R228* alleles should retain some level of avirulence activity. We had observed that, in some experiments, strains expressing the nonsense avrRpt2 alleles occasionally grew to levels slightly lower than virulent $P$. syringae pv. tomato DC3000 on resistant plants expressing a functional RPS2 gene (Fig. 1B). It is possi- ble that the nonsense avrRpt 2 alleles retain some avirulence activity but that, in the context of pathogen infection, this activity is not sufficient to activate defenses rapidly or strongly enough, or both, to prevent $P$. syringae pv. tomato DC3000 from growing to high levels and causing disease. We hypothesized that this residual avirulence activity might be more readily detectable if the two mutant AvrRpt2 proteins were overexpressed from within the plant. To express AvrRpt2 at high levels in planta, we used Arabidopsis lines expressing either wildtype avrRpt 2 or the two nonsense alleles under the control of a DEX-inducible promoter (Axtell et al. 2001; McNellis et al. 1998). McNellis and associates (1998) previously demonstrated that application of increasing concentrations of DEX to plants expressing wild-type $a v r R p t 2$ results in a corresponding increase in AvrRpt 2 protein. Furthermore, they showed that this expression can recapitulate the effects of bacterial delivery of AvrRpt2, resulting in tissue collapse indicative of the HR (McNellis et al. 1998).

As summarized in Table 1, inoculation with $25 \mu \mathrm{M}$ DEX induced rapid tissue collapse within $12 \mathrm{~h}$ postinfiltration (hpi) in RPS2 Arabidopsis plants expressing wild-type AvrRpt2. DEX-induced tissue collapse was also observed in plants expressing the W200* and R228* mutants but appeared to be slightly delayed compared with plants expressing wild-type AvrRpt2 (Table 1). However, by 30 hpi, the majority of the leaves infiltrated with DEX had undergone complete tissue collapse, both in lines expressing wild-type avrRpt 2 and those expressing the nonsense alleles (data not shown). The tissue collapse was dependent on the presence of both avrRpt 2 and $R P S 2$, as no collapse was apparent in leaves of transgenic $R P S 2$ plants expressing an empty vector or of transgenic plants expressing DEX-inducible avrRpt2 in an rps 2 mutant background (Table 1). Therefore, when expressed at high levels inside the plant, the two C-terminally truncated AvrRpt2 mutants encoded by the $\mathrm{W} 200^{*}$ and $\mathrm{R} 228^{*}$ alleles are able to
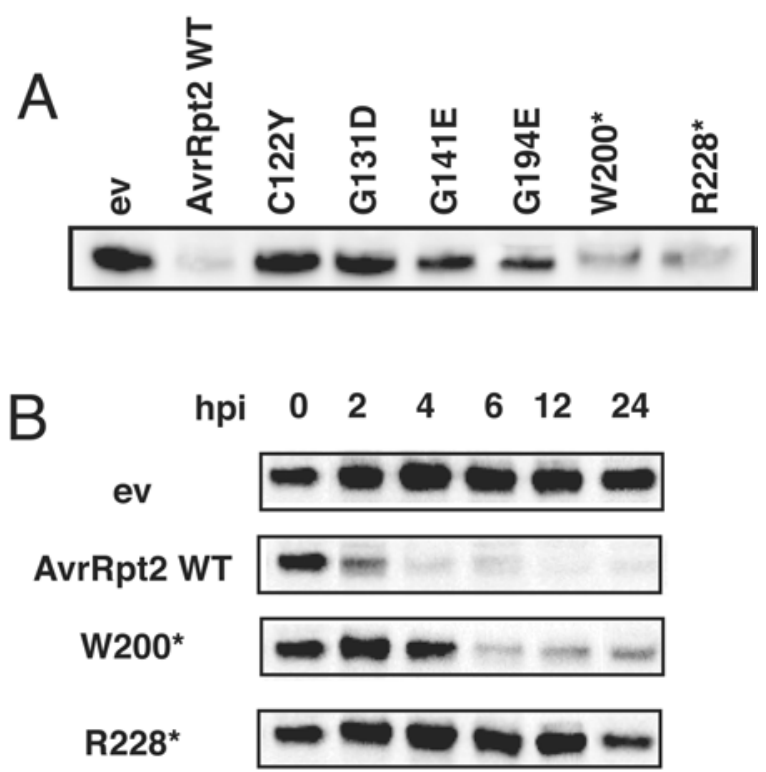

Fig. 4. Two C-terminal deletion mutants of AvrRpt 2 retain the ability to induce the disappearance of RIN4. Blots of total protein extracts from plants infected with Pseudomonas syringae pv. tomato DC3000 carrying an empty vector (ev), wild-type (WT), or mutant avrRpt2 alleles, reacted with antiRIN4 antibody. A, Arabidopsis Col-0 rps2 leaves were syringe-inoculated with bacterial suspensions of $5 \times 10^{8} \mathrm{CFU} / \mathrm{ml}$, and leaves were harvested 6.5 $\mathrm{h}$ after inoculation. B, Arabidopsis Col-0 rsp2 leaves were inoculated with bacterial suspensions of $5 \times 10^{7} \mathrm{CFU} / \mathrm{ml}$, and leaves were harvested at several timepoints after inoculation, as indicated. hpi $=$ hours postinoculation. These experiments were repeated three times with similar results. 
induce RPS2-dependent cell death. These observations are consistent with the hypothesis that these avrRpt 2 mutants possess residual avirulence activity. The fact that transgenic plants expressing the W200* and R228* alleles were initially isolated in a screen for mutations that abolish RPS2-dependent cell death (Axtell et al. 2001) can probably be attributed to the lower levels of DEX (10 to $20 \mathrm{nM}$ ) used to express the avrRpt 2 transgene in the screen.

\section{All avrRpt 2 mutants fail to interfere with the RPM1-mediated hypersensitive response.}

RIN4 is required for the activation of RPMI-mediated defense responses (Mackey et al. 2002). Accordingly, Arabidopsis plants with reduced levels of RIN4 fail to develop a HR in response to infection with $P$. syringae expressing avrRpml (Mackey et al. 2002). Consistent with its ability to induce the disappearance of RIN4, AvrRpt2 suppresses the RPM1-dependent HR (Table 2) (Ritter and Dangl 1996). We predicted that the ability of the avrRpt 2 mutant alleles to suppress the $R P M 1$-dependent HR would be correlated with their ability to trigger the disappearance of RIN4. As expected, expression of the C122Y, G131D, G141E and G194E alleles that fail to induce the disappearance of RIN4 also failed to suppress the $R P M 1$-dependent HR (Table 2). However, P. syringae expressing avrRpm1 and either the W200* or the R228* mutants also failed to suppress the RPMI-dependent HR on Arabidopsis (Table 2). Initially, these results may seem inconsistent with the ability of the W200* and R228* mutants to trigger RIN4 elimination (Fig. 3A). However, this apparent discrepancy might be explained if the delay in RIN4 elimination in response to infection with $P$. syringae expressing the W200* and R228* mutants (Fig. 3B) allows sufficient RIN4 protein to be present early in the interaction to result in activation of $R P M 1$ mediated defense signaling and, thus, induction of the RPMImediated HR.

\section{DISCUSSION}

\section{AvrRpt 2 mutants do not undergo efficient host-dependent processing.}

During infection, AvrRpt 2 is synthesized by P. syringae and then is translocated via the TTSS into the host cell, where the protein functions either to promote disease on susceptible plants or to elicit host defenses on resistant plants. Due to the complexity of this process, the function of AvrRpt 2 could be easily disrupted by mutations that interfere with any aspect of expression or delivery of the protein. Thus, to facilitate our

Table 1. Two C-terminal deletion mutants of AvrRpt2 retain the ability to elicit RPS2-dependent tissue collapse when overexpressed in planta

\begin{tabular}{lccc}
\hline & \multicolumn{3}{c}{ Number of leaves responding by $^{\mathbf{b}}$} \\
\cline { 2 - 4 } Plant genotype $^{\mathbf{a}}$ & $\mathbf{8 ~ h p i}$ & $\mathbf{1 2} \mathbf{~ h p i}$ & $\mathbf{2 4} \mathbf{~ h p i}$ \\
\hline RPS2/ev & $0 / 11$ & $0 / 11$ & $0 / 11$ \\
rps2/avrRpt2 & $0 / 12$ & $0 / 12$ & $0 / 12$ \\
RPS2/avrRpt 2 & $2 / 10$ & $8 / 10$ & $10 / 10$ \\
RPS2/W200* & $1 / 12$ & $6 / 12$ & $9 / 12$ \\
RPS2/R228* & $2 / 11$ & $5 / 11$ & $7 / 11$ \\
\hline
\end{tabular}

${ }^{a}$ Wild-type avrRpt2 was overexpressed in either the rps 2 or RPS2 Col-0 Arabidopsis. The W200* and R228* alleles were both expressed in the RPS2 background. ev refers to the T-DNA insert not containing avrRpt2. All alleles were expressed under the control of a dexamethasone (DEX)-inducible promoter (Axtell et al. 2001; McNellis et al. 1998).

${ }^{\mathrm{b}}$ Leaves were scored at 8,12 , and $24 \mathrm{~h}$ postinfiltration (hpi) with DEX. Numbers indicate leaves showing RPS2-dependent tissue collapse/total number of leaves scored. Similar results were obtained in three independent experiments. analysis of the AvrRpt 2 mutants, we first needed to identify those mutations that disrupt the function of AvrRpt 2 in planta rather than those that interfere with protein stability or translocation into the plant cell. For the four AvrRpt 2 missense mutants that lack significant virulence and avirulence activities, we needed to rely on immuno-detection of the processed AvrRpt 2 protein to determine whether the mutant proteins are translocated into the plant cell. However, as AvrRpt 2 encoded by the missense alleles were either unstable in $P$. syringae or did not undergo efficient host-dependent processing, we were unable to determine whether these proteins are translocated into the plant cell (Fig. 3). Nevertheless, when overexpressed at high levels in planta AvrRpt 2 encoded by the C122Y/G131D and G141E alleles failed to elicit RPS2-dependent tissue collapse (data not shown), suggesting that these mutants have lost avirulence activity.

The significance of AvrRpt2 processing and the mechanism underlying this activity are not understood. In the case of AvrPphB, another TTSS effector of $P$. syringae that undergoes processing, this event occurs both in the bacteria and in the host and appears to result from an autocatalytic protease activity (Puri et al. 1997; Shao et al. 2002). In addition to being directly required for autocatalysis, this protease activity is required for the avirulence activity of AvrPphB (Shao et al. 2002). The inefficient processing of AvrRpt 2 encoded by the four nonfunctional missense mutants might suggest that AvrRpt 2 must also be processed to be active. Thus, as for AvrPphB, the absence of processing of the proteins encoded by the missense mutants might be indicative of the loss of a protease activity required for the avirulence function of AvrRpt2, as proposed by Axtell and associates (2003). Alternatively, the missense mutations might simply disrupt the structure of the proteins, possibly rendering them unsuitable substrates for processing by another protease within the plant. The C-terminal deletion mutants of AvrRpt 2 encoded by the two nonsense alleles do not appear to be processed when overexpressed in planta (Fig. 3B). However, as the Cterminal deletion mutants retain both virulence activity and the ability to trigger RIN4 elimination, it is clear that some functional protein is translocated into the plant cell. Given that the full-length forms of these mutant proteins appear to be less stable than wild-type AvrRpt2 (Fig. 3), it is possible that they are processed in planta, but that the processed forms are also unstable and, thus, not easily detected by protein gel-blot analysis. Interestingly, the W200* mutation truncates AvrRpt 2 before the His 208 residue predicted to be essential for the putative cysteine protease activity of the protein and for its ability to induce RIN4 elimination (Axtell et al. 2003). Thus, our finding that the W200* mutant retains the ability to

Table 2. Ability of avrRpt2 mutant alleles to suppress the RPM1dependent hypersensitive response (HR)

\begin{tabular}{lc}
\hline avr genes & Number of leaves showing $\mathbf{H R}^{\mathbf{a}}$ \\
\hline none & $0 / 20$ \\
avrRpm1 & $20 / 20$ \\
avrRpm1; avrRpt2 WT & $0 / 23$ \\
avrRpm1; avrRpt2 C122Y & $10 / 10$ \\
avrRpm1; avrRpt2 G131D & $9 / 10$ \\
avrRpm 1; avrRpt2 G141R & $12 / 14$ \\
avrRpm1; avrRpt2 G194E & $10 / 11$ \\
avrRpm1; avrRpt2 W200* & $12 / 12$ \\
avrRpm1; avrRpt2 R228* & $10 / 12$ \\
\hline
\end{tabular}

${ }^{a}$ Pseudomonas syringae pv. maculicola M4 alone or carrying the avr genes indicated was inoculated on Arabidopsis Col-0 rps2. The HR, visible as complete collapse of the inoculated leaf region, was scored 5.5 $\mathrm{h}$ after inoculation. Similar results were observed in three independent experiments. 
both promote $P$. syringae pv. tomato DC3000 virulence (Fig. 2) and to induce RIN4 elimination (Fig. 4) suggests that the putative cysteine protease activity is not required for either AvrRpt2 function. However, the discrepancy between our results and those of Axtell and associates could be due to the different gene expression systems and hosts used in the two studies (i.e., Agrobacterium-mediated transient expression in tobacco used by Axtell and associates).

\section{C-terminal deletions of AvrRpt 2 exhibit reduced efficiency in inducing disappearance of RIN4.}

We found that while the C-terminal deletion mutants encoded by the W200* and R228* alleles retained the ability to induce the disappearance of RIN4 when delivered by $P$. syringae pv. tomato DC3000, they did so with reduced efficiency compared with wild-type AvrRpt2 (Fig. 4B). Moreover, the two C-terminal deletion mutants were able to trigger RPS2-specific cell death only when expressed at high levels in planta (Table 1). These observations are consistent with the phenotypes of Arabidopsis lines overexpressing RIN4, in which a delay in the AvrRpt2-induced reduction of RIN4 levels is correlated with impaired RPS2-dependent resistance responses (Mackey et al. 2003). Thus, our results provide additional support for the hypothesis that the disappearance of RIN4 leads to activation of RPS2-mediated resistance (Axtell and Staskawicz 2003; Mackey et al. 2003).

It is intriguing that, despite exhibiting residual avirulence activity, the two C-terminal deletion mutants of AvrRpt 2 do not elicit effective resistance in RPS2 plants when delivered by $P$. syringae pv. tomato DC3000. Since the two C-terminal deletion mutants appear to be less stable than wild-type AvrRpt2 (Fig. 3A), it is possible that the level to which they accumulate inside the plant cell after delivery by the bacteria is not sufficient to activate RPS2-dependent defenses. Alternatively, the mutants may be able to activate RPS2dependent defenses but are unable to do so quickly enough to prevent pathogen growth and disease. The latter hypothesis is consistent with the results of gene expression profiling experiments that reveal that, although similar changes in host gene expression occur during resistant and susceptible interactions between $P$. syringae and Arabidopsis, these changes occur more slowly during susceptible interactions (Tao et al. 2003).

\section{A decrease in virulence is correlated}

\section{with reduced efficiency of RIN4 elimination.}

Although several $P$. syringae TTSS effector proteins have been shown to play a role in virulence, little is known about their mode of action (Guttman et al. 2002; Leach et al. 2001). It has been proposed that AvrRpt 2 exerts its virulence activity by modifying a RIN4-containing defense activator (Mackey et al. 2003). We demonstrate that the reduction in the virulence activity of the AvrRpt 2 mutants is correlated with reduced efficiency in triggering the disappearance of RIN4. This result is consistent with the hypothesis that the virulence activity of AvrRpt 2 is mediated through the removal of a RIN4-containing complex. However, the role of RIN4 in basal defense against $P$. syringae is unclear, and the importance of RIN4 elimination in AvrRpt2 virulence has not been explored (discussed below). Thus, it is possible that the disappearance of RIN4 does not play a direct role in the virulence activity of AvrRpt2. For example, AvrRpt2 could act through a RIN4-independent mechanism by modifying one or more other virulence targets in the plant cell. In this scenario, the apparent reduction in stability of the two AvrRpt2 C-terminal deletion mutants (Fig. 3) could account for the partial reduction in their virulence activity.

\section{C-terminal deletion of AvrRpt2}

dissociates its virulence and avirulence activities.

The introgression of $R$ genes into crop plants remains an important strategy for generating resistant cultivars (McDowell and Woffenden 2003; Pink 2002). Unfortunately, the durability of this type of resistance in the field is short-lived, due to selection for mutations in the pathogen population that abolish the activity of avirulence determinants (Leach et al. 2001). Such changes are often observed despite the fitness penalty that might be imposed on the pathogen due to the loss of activity of $a v r$ genes that also contribute to virulence. The relatively common occurrence of such changes might suggest that avirulence determinants are generally tolerant of mutations that disrupt their avirulence activity but that only minimally affect their contribution to virulence. Accordingly, mutations that result in a loss of avirulence function but not of virulence activity have been identified in two bacterial avirulence genes, avrPto from P. syringae pv. tomato (Shan et al. 2000) and avrBs2 from Xanthomonas campestris pv. vesicatoria (Gassmann et al. 2000). In the case of avrBs $2, X$. campestris pv. vesicatoria strains expressing avrBs 2 mutant alleles that differentially affect the virulence and avirulence activities of AvrBs 2 were able to cause disease on pepper plants expressing the cognate resistance gene $B s 2$ in the field. This indicates that $a v r B s 2$ can evolve to avoid recognition by plants expressing $B s 2$ while maintaining virulence activity. In this study, we show that the two C-terminal deletion mutants of AvrRpt2, W200*, and $\mathrm{R} 228 *$ are severely impaired in triggering RPS2-dependent resistance but retain the ability to promote the virulence of $P$. syringae pv. tomato DC3000 on susceptible plants lacking a functional RPS2 gene. Thus, we provide another example of specific mutations in a bacterial avirulence protein allowing the pathogen to escape $R$-gene-mediated host recognition without incurring a significant loss in virulence.

Although a role in virulence has been identified for several avirulence genes, little is known about the mechanistic relationships between their dual activities. This question is highly relevant to the ongoing debate regarding whether the products of $a v r$ genes interact directly with the products of their cognate $R$ genes or, alternatively, whether the interaction is indirect, as postulated by the guard hypothesis (Dangl and Jones 2001; Van der Biezen and Jones 1998). In the case of AvrPto, point mutations were identified that disrupt the interaction of the protein with the product of the $R$ gene Pto but that do not affect the virulence activity of AvrPto. This result has been interpreted as an indication of distinct structural requirements for the two activities of AvrPto (Shan et al. 2000). The two nonsense mutations of AvrRpt 2 also differentially affect its virulence and avirulence activities. However, the reduced efficiency of the C-terminal deletion mutants in triggering RIN4 elimination suggests an alternative explanation to structural uncoupling for the functional separation of these two activities. We envision that, if a RIN4-containing complex is the only cellular target of AvrRpt2, then all of the activities that we are able to assay for AvrRpt2 (virulence, avirulence, and interference with $R P M 1$-mediated resistance) could be modulated to a different degree by the function of the components of this complex. Thus, the effect that mutations in AvrRpt2 would have on each of its activities would depend on the extent to which these mutations impair the ability of AvrRpt 2 to trigger RIN4 elimination. For example, we show that the two AvrRpt 2 C-terminal deletion mutants (W200* and R228*) fail to interfere with the RPM1-dependent HR but still retain the ability to promote the growth of $P$. syringae on susceptible plants. This could be because the two C-terminal deletion mutants do not eliminate RIN4 quickly enough to prevent activation of RPM1 signaling (an early event in the interaction) but 
eliminate enough of a RIN4-containing defense activator over the course of the infection to allow $P$. syringae pv. tomato to proliferate and cause disease on No-0 rps2. Thus, our analysis of the phenotypes of the two C-terminal deletion mutants of AvrRpt 2 and the possible involvement of RIN4 in all aspects of AvrRpt 2 activity suggest that we need not invoke structural uncoupling as the basis of the functional uncoupling of the various activities of AvrRpt2. This may be a common feature of other virulence/avirulence factors that work via a mechanism similar to AvrRpt2. The role of RIN4 in defense against bacterial infection is unclear and needs to be addressed as we consider the hypothesis that RIN4 is a target of virulence factors such as AvrRpt2. A more thorough understanding of these questions awaits the analysis of a rin4 null mutant (Mackey et al. 2003).

\section{MATERIALS AND METHODS}

\section{Bacterial strains and plasmids.}

The bacterial pathogen Pseudomonas syringae pv. tomato DC3000 has been described previously (Whalen et al. 1991). Plasmid pV288, which carries wild-type avrRpt2 on plasmid pVSP61 (Whalen et al. 1991), was used as positive control in all experiments. P. syringae pv. tomato DC3000 not carrying avrRpt2 harbored the empty vector pVSP61 (Whalen et al. 1991). For assaying the ability of AvrRpt 2 to interfere with the RPM1-dependent HR, $P$. syringae pv. maculicola M4 and $P$. syringae pv. maculicola carrying avrRpml were used (Debener et al. 1991). All new constructs generated in this study (discussed below) were introduced into $P$. syringae strains by triparental mating using helper plasmid pRK2013 (Figurski and Helinski 1979).

\section{Plant material, growth conditions, and inoculation.}

A. thaliana ecotypes Col-0 and No-0 were used. The generation of Col-0 and No-0 lines carrying the rps2-201C mutation has been described previously (Chen et al. 2000; Kunkel et al. 1993). A. thaliana plants were maintained in growth chambers under an 8 -h photoperiod at $21^{\circ} \mathrm{C}$ and $75 \%$ humidity. Disease and in planta growth assays were carried out on 3- to 4-weekold plants that were inoculated by dipping in a bacterial suspension containing the surfactant Silwett L-77 at a concentration of $0.02 \%$ (Kunkel et al. 1993). The total number of bacteria in infected leaves was monitored according to Tornero and Dangl (2001) with the following modifications: four leaves (two from each of two infected plants) were shaken for $1 \mathrm{~h}$ in $1 \mathrm{ml}$ of $10 \mathrm{mM} \mathrm{MgCl}_{2}, 0.02 \%$ Silwett at $30^{\circ} \mathrm{C}$. The bathing solution was then serially diluted and plated, as described by Tornero and Dangl (2001). For the HR assay, a 1-ml flattipped syringe was used to infiltrate leaves with a bacterial suspension at $1 \times 10^{7} \mathrm{CFU}$ of $P$. syringae pv. tomato per $\mathrm{ml}$ (Kunkel et al. 1993) or $5 \times 10^{7} \mathrm{CFU}$ of $P$. syringae pv. maculicola per ml (Ritter and Dangl 1996). Arabidopsis lines expressing wild-type or mutant avrRpt 2 alleles under the control of a DEX-inducible promoter have been described previously (Axtell et al. 2001; McNellis et al. 1998)). The transgenic lines analyzed in this study contained avrRpt 2 alleles with mutations resulting in the following amino acid mutations: C122Y/G131D, G141E, G194E, W200*, or R228* (Axtell et al. 2001). For DEX treatment, leaves were infiltrated with 25 $\mu \mathrm{M}$ DEX (Sigma, St. Louis) using a 1-ml flat-tipped syringe.

\section{Generation of avrRpt2 mutants.}

Mutations were introduced into the avrRpt 2 coding sequence by site-directed mutagenesis (Kunkel 1985). A singlestranded uracil-containing template was prepared using the plasmid pRSR0 (Innes et al. 1993). Following mutagenesis, the presence of the mutations introduced in the avrRpt 2 coding region was confirmed by sequencing. Following digestion with EcoRI and HindIII, a 1.4-kb DNA fragment encompassing the avrRpt 2 open reading frame and its native promoter was subcloned from the mutagenized pRSR0 plasmids into pVSP61.

\section{Protein extraction and immunoblot analysis.}

Following treatment with either bacteria or DEX, leaves were flash-frozen in liquid nitrogen and were stored at $-80^{\circ} \mathrm{C}$ until they were subject to protein extraction. Total protein was extracted from Arabidopsis leaves, according to Nimchuk and associates (2000). Leaves (3 to 4) were ground in $150 \mu \mathrm{l}$ of extraction buffer. Protein concentrations were measured with the Bio-Rad protein assay kit (Bio-Rad, Hercules, CA, U.S.A.) and were used to equalize the loading of lanes on sodium dodecyl sulfate polyacrylamide gels. Equal loading was confirmed by Ponceau-S staining, following the transfer of proteins to nitrocellulose membranes. The presence of AvrRpt2 was detected by protein gel blot analysis, using affinity purified polyclonal anti-AvrRpt 2 antibody, generated in our laboratory. RIN4 protein was detected using polyclonal anti-RIN4 antibody (Mackey et al. 2002). Western blot analyses were performed according to standard protocols (Ausubel et al. 1987).

\section{ACKNOWLEDGMENTS}

We thank W. Gassmann, Z. Chen, J. Agnew, and D. Brooks for helpful comments and discussion of the manuscript. We also thank Y. Belkhadir and J. Dangl for anti-RIN4 sera and M. Axtell and B. Staskawicz for the transgenic Arabidopsis lines containing the DEX-inducible avrRpt2 alleles. This work was supported by United States Department of Agriculture grant 2002-35319-12729 and a David and Lucille Packard Fellowship for Science and Engineering. Funds to cover publication costs were provided by the Jean Lowenhaupt Botany Fund.

\section{LITERATURE CITED}

Ausubel, F. M., Brent, R., Kingston, R. E., Moore, D. D., Seidman, J. G., Smith, J. A., and Struhl, K. 1987. Current Protocols in Molecular Biology. Wiley-Interscience, New York.

Axtell, M. J., and Staskawicz, B. J. 2003. Initiation of RPS2-specified disease resistance in Arabidopsis is coupled to the AvrRpt2-directed elimination of RIN4. Cell 112:369-377.

Axtell, M. J., McNellis, T. W., Mudgett, M. B., Hsu, C. S., and Staskawicz, B. J. 2001. Mutational analysis of the Arabidopsis RPS2 disease resistance gene and the corresponding Pseudomonas syringae avrRpt2 avirulence gene. Mol. Plant-Microbe Interact. 14:181-188.

Axtell, M. J., Chisholm, S. T., Dahlbeck, D., and Staskawicz, B. J. 2003. Genetic and molecular evidence that the Pseudomonas syringae type III effector protein AvrRpt2 is a cysteine protease. Mol. Microbiol. 49:1537-1546.

Chen, Z, Kloek, A. P., Boch, J., Katagiri, F., and Kunkel, B. N. 2000. The Pseudomonas syringae avrRpt2 gene product promotes pathogen virulence from inside plant cells. Mol. Plant-Microbe Interact. 13:13121321.

Dangl, J. L., and Jones, J. D. 2001. Plant pathogens and integrated defense responses to infection. Nature 411:826-833.

Debener, T., Lehnackers, H., Arnold, M., and Dangl, J. 1991. Identification and molecular mapping of a single Arabidopsis thaliana locus determining resistance to a phytopathogenic Pseudomonas syringae isolate. Plant J. 1:289-302.

Figurski, D., and Helinski, D. R. 1979. Replication of an origin-containing derivative of plasmid RK2 is dependent on a plasmid function provided in trans. Proc. Natl. Acad. Sci. U.S.A. 76:1648-1652.

Galan, J. E., and Collmer, A. 1999. Type III secretion machines: Bacterial devices for protein delivery into host cells. Science 284:1322-1328.

Gassmann, W., Dahlbeck, D., Chesnokova, O., Minsavage, G. V., Jones, J. B., and Staskawicz, B. J. 2000. Molecular evolution of virulence in natural field strains of Xanthomonas campestris pv. vesicatoria. J. Bacteriol. 182:7053-7059.

Greenberg, J. T. 1997. Programmed cell-death in plant pathogen interactions. Annu. Rev. Plant. Physiol. Plant Mol. Biol. 48:525-545.

Guttman, D. S., and Greenberg, J. T. 2001. Functional analysis of the type 
III effectors AvrRpt2 and AvrRpm1 of Pseudomonas syringae with the use of a single-copy genomic integration system. Mol. Plant-Microbe Interact. 14:145-155.

Guttman, D. S., Vinatzer, B. A., Sarkar, S. F., Ranall, M. V., Kettler, G. and Greenberg, J. T. 2002. A functional screen for the type III (Hrp) secretome of the plant pathogen Pseudomonas syringae. Science 295:1722-1726.

Innes, R. W., Bent, A. F., Kunkel, B. N., Bisgrove, S. R., and Staskawicz, B. J. 1993. Molecular analysis of avirulence gene avrRpt2 and identification of a putative regulatory sequence common to all known Pseudomonas syringae avirulence genes. J. Bacteriol. 175:4859-4869.

Kunkel, B. N., Bent, A. F., Dahlbeck, D., Innes, R. W., and Staskawicz, B J. 1993. RPS2, an Arabidopsis disease resistance locus specifying recognition of Pseudomonas syringae strains expressing the avirulence gene avrRpt2. Plant Cell. 5:865-875.

Kunkel, T. A. 1985. Rapid and efficient site-specific mutagenesis without phenotypic selection. Proc. Natl. Acad. Sci. U.S.A. 82:488-492.

Leach, J. E., Vera Cruz, C. M., Bai, J., and Leung, H. 2001. Pathogen fitness penalty as a predictor of durability of disease resistance genes. Annu. Rev. Phytopathol. 39:187-224.

Leister, R. T, Ausubel, F. M., and Katagiri, F. 1996. Molecular recognition of pathogen attack occurs inside of plant cells in plant disease resistance specified by the Arabidopsis genes RPS2 and RPM1. Proc. Natl. Acad. Sci. U.S.A. 93:15497-15502

Mackey, D., Holt, B. F., Wiig, A., and Dangl, J. L. 2002. RIN4 interacts with Pseudomonas syringae type III effector molecules and is required for RPM1-mediated resistance in Arabidopsis. Cell 108:743-754.

Mackey, D., Belkhadir, Y., Alonso, J. M., Ecker, J. R., and Dangl, J. L. 2003. Arabidopsis RIN4 is a target of the type III virulence effector AvrRpt2 and modulates RPS2-mediated resistance. Cell 112:379-389.

McDowell, J. M., and Woffenden, B. J. 2003. Plant disease resistance genes: Recent insights and potential applications. Trends Biotech. 21:178-183

McNellis, T. W., Mudgett, M. B., Li, K., Aoyama, T., Horvath, D., Chua, N.-H., and Staskawicz, B. J. 1998. Glucocorticoid-inducible expression of a bacterial avirulence gene in transgenic Arabidopsis induced hypersensitive cell death. Plant J. 14:247-357.

Mudgett, M. B., and Staskawicz, B. J. 1999. Characterization of the Pseudomonas syringae pv. tomato AvrRpt2 protein: Demonstration of secretion and processing during bacterial pathogenesis. Mol. Microbiol. 32:927-941.
Nimchuk, Z., Marios, E., Kjemtrup, S., Leister, R. T., Katagiri, F., and Dangl, J. L. 2000. Eukaryotic fatty acylation drives plasma membrane targeting and enhances function of several type III effector proteins from Pseudomonas syringae. Cell. 101:353-363.

Pink, D. A. C. 2002. Strategies using genes for non-durable resistance. Euphytica. 124:227-236.

Puri, N., Jenner, C., Bennett, M., Stewart, R., Mansfield, J., Lyons, N., and Taylor, J. 1997. Expression of avrPphB, an avirulence gene from Pseudomonas syringae pv. phaseolicola, and the delivery of signals causing the hypersensitive reaction in bean. Mol. Plant-Microbe Interact. 10:247-256.

Ritter, C., and Dangl, J. L. 1996. Interference between two specific pathogen recognition events mediated by distinct plant disease resistance genes. Plant Cell 8:251-257.

Shan, L., He, P., Zhou, J. M., and Tang, X. 2000. A cluster of mutations disrupt the avirulence but not the virulence function of AvrPto. Mol. Plant-Microbe Interact. 13:592-8.

Shao, F., Merritt, P. M., Bao, Z. Q., Innes, R. W., and Dixon, J. E. 2002. A Yersinia effector and a Pseudomonas avirulence protein define a family of cysteine proteases functioning in bacterial pathogenesis. Cell 109:575-588.

Tao, Y., Xie, Zhiyi, Chen, W., Glazebrook, J., Chang, H.-S., Han, B., Zhu, T., Zou, G., and Katagiri, F. 2003. Quantitative nature of Arabidopsis responses during compatible and incompatible interactions with the bacterial pathogen Pseudomonas syringae. Plant Cell 15:317-330.

Tornero, P., and Dangl, J. L. 2001. A high-throughput method for quantifying growth of phytopathogenic bacteria in Arabidopsis thaliana. Plant J. 28:475-481.

Van Der Biezen, E. A., and Jones, J. D. G. 1998. Plant disease-resistance proteins and the gene-for-gene concept. Trends Biochem. 23:454-456.

Whalen, M., Innes, R., Bent, A., and Staskawicz, B. 1991. Identification of Pseudomonas syringae pathogens of Arabidopsis thaliana and a bacterial gene determining avirulence on both Arabidopsis and soybean. Plant Cell 3:49-59.

Wu, Y., Wood, M. D., Tao, Y., and Katagiri, F. 2003. Direct delivery of bacterial avirulence proteins into resistant Arabidopsis protoplasts leads to hypersensitive cell death. Plant J. 33:131-137.

Yu, G. L., Katagiri, F., and Ausubel, F. M. 1993. Arabidopsis mutations at the RPS2 locus result in loss of resistance to Pseudomonas syringae strains expressing the avirulence gene avrRpt2. Mol. Plant-Microbe Interact. 6:434-443. 